\title{
miRNA expression profiles in cerebrospinal fluid and blood of patients with Alzheimer's disease and other types of dementia - an exploratory study
}

Sofie Sølvsten Sørensen ${ }^{1}$, Ann-Britt Nygaard ${ }^{2}$ and Thomas Christensen ${ }^{1 *}$

\begin{abstract}
Background: MicroRNAs (miRNAs) are small non-coding RNA molecules that function as posttranscriptional regulators of gene expression. Measurements of miRNAs in cerebrospinal fluid (CSF) and blood have just started gaining attention as a novel diagnostic tool for various neurological conditions. The purpose of this exploratory investigation was to analyze the expression of miRNAs in CSF and blood of patients with Alzheimer's disease (AD) and other neurodegenerative disorders in order to identify potential miRNA biomarker candidates able to separate AD from other types of dementia.

Methods: CSF was collected by lumbar puncture performed on 10 patients diagnosed with AD and 10 patients diagnosed with either vascular dementia, frontotemporal dementia or dementia with Lewy bodies. Blood samples were taken immediately after. Total RNA was extracted from cell free fractions of CSF and plasma, and a screening for 372 known miRNA sequences was carried out by real time quantitative polymerase chain reactions (miRCURY LNA $^{\text {TM }}$ Universal RT miRNA PCR, Polyadenylation and cDNA synthesis kit, Exiqon).

Results: Fifty-two miRNAs were detected in CSF in at least nine out of ten patients in both groups. Among these, two miRNAs (let-7i-5p and miR-15a-5p) were found significantly up-regulated and one miRNA (miR-29c-3p) was found significantly down-regulated in patients with AD compared to controls. One hundred and sixty-eight miRNAs were frequently detected in the blood, among which miR-590-5p and miR-142-5p were significantly up-regulated and miR-194-5p was significantly down-regulated in AD patients compared to controls.

Conclusions: Detection of miRNA expression profiles in blood and in particular CSF of patients diagnosed with different types of dementia is feasible and it seems that several expressional differences between AD and other dementia types do exist when measured in a clinically relevant setup. In this explorative pilot study, the deregulated miRNAs in CSF of AD patients may be associated with relevant target genes related to AD pathology, including APP and BACE1, which suggests that miRNAs are interesting candidates for AD biomarkers in the future.
\end{abstract}

Keywords: Alzheimer's disease, Dementia, Neurodegenerative disease, miRNA expression, Diagnostic biomarker, Cerebrospinal fluid, Blood

\footnotetext{
*Correspondence: thoc@regionh.dk

'Department of Neurology, Copenhagen University Hospital, Nordsjællands

Hospital, Dyrehavevej 29, 3400 Hillerød, Denmark

Full list of author information is available at the end of the article
} 


\section{Background}

Alzheimer's disease (AD) is an age-related progressive neurodegenerative disorder characterized by cognitive impairment and neuropsychiatric symptoms leading to restrictions in the activities of daily living. AD is the most common type of dementia accounting for 50-70\% of all dementia cases. Approximately $10 \%$ of elderly people (65+ years) are affected by AD with an age-specific prevalence that almost doubles every five years after 65 [1]. Because of the ageing population worldwide, the increasing incidence and socioeconomic impact of $\mathrm{AD}$ and other dementias represents a growing challenge to public health all over the world $[2,3]$.

The complex neurobiology of AD, which is not fully understood, includes the development of extracellular amyloid plaques and intracellular neurofibrillary tangles, vascular changes, neuronal inflammation, neurochemical changes, and progressive brain atrophy [4-7].

The prospect of getting an effective treatment is not only complicated by the lack of knowledge about the underlying pathophysiological mechanisms of $\mathrm{AD}$ but also by the difficulty of accurately diagnosing $\mathrm{AD}$ at an early stage.

When compared to healthy individuals, existing cerebrospinal fluid (CSF) biomarkers support the clinical diagnosis of $\mathrm{AD}$ with a high predictive accuracy, however, in differentiating $\mathrm{AD}$ from other types of dementia (e.g. vascular dementia, frontotemporal dementia and dementia with Lewy bodies) the current biomarkers have their limitations. In a newly published meta-analysis the sensitivity and specificity of CSF amyloid beta (A $\beta 1-42)$ for separating $\mathrm{AD}$ from other dementias were estimated to be 73 and $67 \%$, respectively [8]. For total CSF tau the sensitivity has been estimated to be $70-75 \%$ and the specificity 74-90\% and for phospo-tau 79-88\% and 78-83\%, respectively, for separating AD from other dementias [9].

Development of new and better biomarkers for $\mathrm{AD}$ and other dementias would result in more accurate diagnoses facilitating the possibility for an early and specialized treatment effort for the growing number of patients with dementia. Especially now that medical treatment options for AD are available, it is particularly important to identify these patients at an early stage.

MicroRNAs (miRNAs) are small non-coding RNA molecules of approximately 22 nucleotides in length that function as posttranscriptional regulators of gene expression. In mammalian cells, miRNAs work through base pairing with complementary sequences within messenger RNA (mRNA) molecules, usually resulting in gene silencing via translational repression. Recent studies indicate that the expression patterns of miRNAs change in relation to various neurological diseases including Alzheimer's disease. Altered expression of specific miRNAs in the brain of $\mathrm{AD}$ patients compared to controls has been reported, including downregulation of $\mathrm{miR}-15 \mathrm{a} / \mathrm{b}, \mathrm{mR}-16$, $\mathrm{miR}-29 \mathrm{a} / \mathrm{b}, \mathrm{miR}-195$, miR-103 and miR-107, which have all been shown to target the $\beta$-site amyloid precursor protein cleaving enzyme 1 (BACE1) involved in the formation of amyloid plaques [10-15]. Lukiw et al. $[16,17]$ studied the miRNA expression in hippocampal tissue of $\mathrm{AD}$ patients, and found upregulation of specific pro-inflammatory miRNAs including miR-9, miR-125b, miR-146a, and miR-155, which all seem to be induced by NF- $\mathrm{B}$, thus indicating a possible role of these miRNAs in neuronal inflammation in $\mathrm{AD}$. On the other hand, Cogswell et al. [18] have found a significant down-regulation of miR-9 in the human hippocampus of AD patients. miR-9 is known from several experimental studies to regulate neuronal differentiation [19].

Measurements of miRNAs in biofluids such as CSF and blood have just started to gain attention as a novel diagnostic tool. Since CSF is separated from blood circulation by the blood-brain barrier (BBB), it makes good biological sense that the CSF could contain unique signatures of miRNA expression specific for various CNS pathologies. Therefore, miRNAs derived from CSF might serve as more valid biomarkers for brain pathologies than those of other body fluids.

To date only a few studies of miRNA expression in CSF of $\mathrm{AD}$ patients have been published. In most of them AD patients were compared to healthy controls, or comparisons of AD patients categorized by different Braak stages were done [18, 20-25]. Even less is known about the differences in CSF miRNA levels between AD and other types of dementia.

Bekris et al. [13] investigated miRNA expression by qPCR in patients with AD compared to both healthy controls and patients with other neurodegenerative diseases. They found miR-15a elevated in the hippocampus and plasma of AD patients where the level was positively correlated with plaque scores. In CSF no expression differences were identified. Burgos et al. [26] profiled miRNAs in CSF and serum by Next Generation Sequencing from patients with AD, Parkinson's disease (PD) and neurologically healthy controls. Numerous deregulated miRNAs between AD and controls in both CSF and serum were found, whereas only a handful of miRNAs was deregulated between patients with $\mathrm{AD}$ and PD. In this study, the expression of several miRNAs in CSF was correlated with Braak stages and tangle scores, including miR-9-3p, for which the level decreased with disease progression. Galimberti et al. [27] profiled miRNAs by qPCR in serum and CSF of AD patients compared to inflammatory and non-inflammatory neurological controls as well as patients with frontotemporal dementia (FTD). They reported down-regulation of miR-125b and miR-26b in CSF of AD patients compared to noninflammatory controls. 
The limited number of studies comparing miRNA CSF levels in $\mathrm{AD}$ patients versus patients with other types of dementia and their opposing results justifies the need for additional studies to investigate the utility of miRNAs as biomarkers in a clinical relevant setup.

The purpose of this exploratory investigation was to analyze the expression of miRNAs by RT-qPCR in CSF and blood from 10 patients diagnosed with Alzheimer's disease and 10 patients diagnosed with either vascular dementia, frontotemporal dementia or dementia with Lewy bodies in order to identify potential miRNA biomarker candidates with the ability to separate AD from other types of dementia using a clinically relevant setup.

\section{Methods}

\section{Patient material and diagnostic evaluation}

Patients who went through diagnostic evaluation at the Memory Clinic, Department of Neurology, Nordsjællands Hospital, from May to December 2014, were asked to participate. All patients gave written and oral informed consent in accordance with the project approval from the Danish Research Ethics Committee (project ID: H-2-2013-069). After completed diagnostic evaluation the study population consisted of 20 patients: one group of $\mathrm{AD}$ patients $(n=10)$, and one control group of patients with other types of dementia, including vascular dementia $(n=4)$, frontotemporal dementia $(n=4)$, and dementia with Lewy bodies $(n=2)$.

Diagnoses were based on patient interviews with supplemental reports from relatives/caregivers, physical and neurological examination, evaluation of psychiatric symptoms (if relevant), assessment of activities of daily living, and cognitive testing by experienced specialist neuropsychologists, whose test collection included MiniMental State Examination (MMSE) and Addenbrooke's Cognitive Examination (ACE). In addition, all patients went through paraclinical testing with standard blood examinations, lumbar puncture and Computer Tomography (CT) scan of the brain. If necessary, the CT scan was supplemented by Magnetic Resonance Imaging (MRI) of the brain to evaluate vascular abnormalities and/or hippocampal atrophy. In addition, all patients underwent functional imaging performed with Fluorodeoxyglucose Positron Emission Tomography $\left({ }^{18} \mathrm{~F}-\mathrm{FDG}-\mathrm{PET}\right)$ to measure glucose metabolism in specific brain areas. Patients with symptoms of dementia with Lewy bodies were scanned with a Dopamine Active Transporter (DAT) scan of the brain to verify low levels of dopamine in the basal ganglia.

Lumbar punctures were done by sterile technique with the use of a $0.7 \mathrm{~mm}$ spinal needle when the patients attended their second visit at the clinic two weeks after referral. At least $7 \mathrm{ml}$ CSF were collected from each patient. Blood samples were drawn immediately after the lumbar puncture. CSF samples were analyzed for glucose, protein, red and white blood cell count, oligoclonal IgG bands, IgG index, total tau, phospo-tau and A $\beta 1-42$ as well as $\mathrm{CSF} /$ serum albumin ratio using the routine tests at the Department of Clinical Biochemistry. At the same time $1 \mathrm{ml} \mathrm{CSF}$ and $1 \mathrm{ml}$ blood from each patient were collected in EDTA tubes for miRNA analysis.

Final diagnoses were made jointly by several specialist neurologists based on all clinical evaluations and paraclinical test results. For the clinical diagnosis of AD the NINCDS-ADRDA criteria for research [28] were used. Only patients who met the criteria for probable AD dementia with evidence of the AD pathophysiological process were included. All patients in the AD group were sporadic $\mathrm{AD}$ patients with a mean age of 70 years. The diagnosis of vascular dementia was made according to the NINDSAIREN criteria [29], and the clinical diagnoses of frontotemporal dementia and dementia with Lewy bodies were made according to the McKhann criteria [30] and McKieth criteria [31], respectively. Table 1 shows the demographic data of the 20 included patients.

\section{Samples for miRNA detection}

For miRNA detection, CSF and blood from each patient were centrifuged immediately after collection and the cellfree fractions were stored at $-80.0{ }^{\circ} \mathrm{C}$. All further sample preparations and experiments were conducted by Exiqon A/S, Denmark. Total RNA was extracted from $200 \mu \mathrm{l} \mathrm{CSF}$ and $200 \mu \mathrm{l}$ plasma using spin column chromatography (miRCURY ${ }^{\mathrm{TM}}$ RNA isolation kit for biofluids).

\section{miRNA real-time qPCR}

Ten microliters of RNA was reverse transcribed in 50- $\mu \mathrm{l}$ reactions using the miRCURY LNA ${ }^{\mathrm{Tm}}$ Universal RT miRNA PCR, Polyadenylation and cDNA synthesis kit (Exiqon). cDNA was diluted 50 times and assayed in 10- $\mu$ PCR reactions. Each miRNA was assayed once by quantitative polymerase chain reactions (qPCR) on the miRNA Readyto-Use PCR, Human panel I containing 372 specific miRNA primers. Negative controls were performed and profiled like the samples. Both RNA (Sp2, Sp4, Sp5, and Sp6) and DNA (Sp3) spike-in controls were added to the panel. Amplification was performed in a LightCycler 480 Real-Time PCR System (Roche). Amplification curves were analyzed using the Roche LC software, both for determination of $\mathrm{Ct}$ values and for melting curve analysis.

\section{Data analysis and normalization}

The amplification efficiency was calculated by using LinReg software. All assays were inspected for distinct temperature melting curves, which were checked to be within known specifications for the assay. Only miRNAs detected with $\mathrm{Ct}<37$ and, in addition, $3 \mathrm{Ct}$ values less than the negative control were included in the data analysis. 
Table 1 Demographic data of the patients included

\begin{tabular}{|c|c|c|c|c|}
\hline Variable & Control group (range) & AD group (range) & Mean difference $(\mathrm{Cl})$ & $p$ \\
\hline Number & 10 & 10 & & \\
\hline Gender (female/male) & $4 / 6$ & $6 / 4$ & & n.s. \\
\hline Age (years) & $69.4(54-79)$ & $70.0(56-82)$ & $0.6(-8.7 ; 9.8)$ & n.s. \\
\hline Duration (years) & $2.8(1.0-5.5)$ & $2.3(1.5-4.0)$ & $-0.5(-1.9 ; 0.9)$ & n.s. \\
\hline MMSE & 24.1 & 21.4 & $-2,7(-8.1 ; 2.7)$ & n.s. \\
\hline ACE & 75.4 & 67.3 & $-8.1(-18.0 ; 1.7)$ & n.s. \\
\hline $\mathrm{A} \beta 1-42$ [pg/ml CSF] & $561.4(315-865)$ & 391.6 (242-755) & $-169.8(-330.0 ;-9.6)$ & 0.04 \\
\hline Total tau $[\mathrm{pg} / \mathrm{ml}$ CSF] & $481.4(228-1200)$ & $540.6(371-742)$ & $59.2(-223.3 ; 341.7)$ & n.s. \\
\hline P-tau $[p g / m l ~ C S F]$ & $60.1(31-148)$ & $82.6(55-110)$ & $22.5(-3.2 ; 48.2)$ & n.s. \\
\hline
\end{tabular}

Control group patients diagnosed with vascular dementia $(n=4)$, frontotemporal dementia $(n=4)$, and dementia with Lewy bodies $(n=2)$. AD group patients all diagnosed with Alzheimer's dementia. MMSE Mini-Mental State Examination score, ACE Addenbrooke's Cognitive Examination score. Mean difference [CI] difference of mean values between groups and $95 \%$ confidence interval of the difference. $p$ p-values of difference calculated by two-sided t-tests or Fisher's exact test (Gender), n.s. nonsignificant $(p>0.05)$

Data that did not pass these criteria were omitted from any further analysis. Thirty-two miRNAs were detected in all CSF samples, and 115 miRNAs were detected in all blood samples. Using NormFinder [32], the best normalizer was found to be the average $\mathrm{Ct}$ value of miRNAs detected in all samples of CSF and blood, respectively, and these global means were used for normalization (relative expression level $=\mathrm{REL}=2^{-\Delta \mathrm{Ct}}$ ) [33]. Both RNA (Sp6) and DNA (Sp3) spike-in controls showed steady levels across all samples indicating accurate $\mathrm{RT}$ reaction and $\mathrm{qPCR}$.

\section{Statistics}

The data of REL or log (REL) did not fit the normal distribution. Therefore, a two-sided non-parametric Mann-Whitney test was performed for statistical analyses (SPSS software) to compare medians (missing values were excluded). $P$ values less than 0.05 were considered significant.

To analyze the predictive power of each miRNA with respect to categorizing patients as having either $\mathrm{AD}$ or another type of dementia, logistic regression analyses with classification accuracies for each miRNA were performed. For this purpose, a leave-one-out (LOO) cross-validation procedure (R Statistics) was applied (missing values were set to zero).

$P$ values for categorical variables were calculated by Fisher's exact test, and $p$ values for comparisons of age, cognitive test score, and CSF routine markers were calculated by student's $t$-test.

Because of the high number of tests performed, all $P$ values were finally corrected for multiple testing with the Benjamini-Hochberg procedure.

\section{Results}

\section{miRNA distribution in body fluids}

Overall, 312 miRNAs were detected in CSF or blood in our study population. Of these, 227 miRNAs occurred in both CSF and blood, 81 miRNAs occurred in blood only, and a minor proportion of 4 miRNAs was found exclusively in the CSF. Among the miRNAs detected in both CSF and blood, some miRNAs were detected predominantly in the CSF and others predominantly in the blood of all patients (Fig. 1)

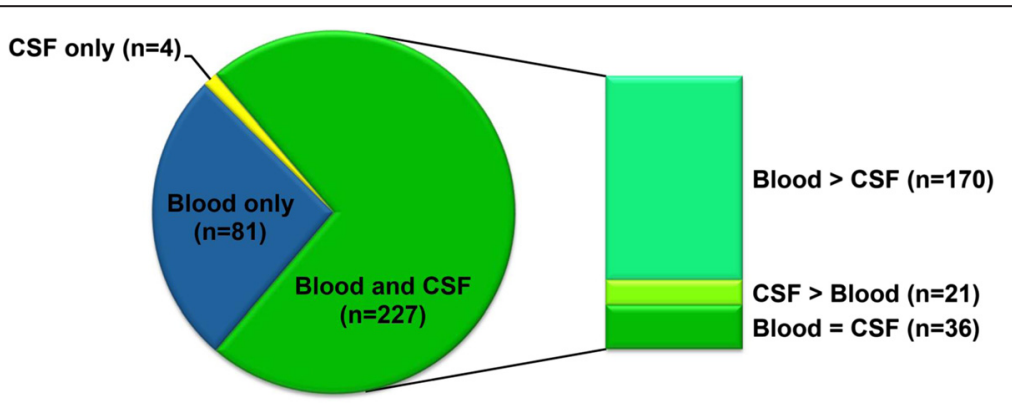

Fig. 1 Distribution of the 312 miRNAs detected in CSF and blood of all patients. Blood > CSF: miRNAs detected more frequently in blood samples than in CSF samples. CSF > blood: miRNAs detected more frequently in CSF samples than in blood samples. Blood=CSF: miRNAs detected with equal frequency in blood and CSF samples 


\section{miRNAs in CSF}

A total of 231 different miRNAs were detected in the CSF. Only 32 different miRNAs were detected in all of the 20 CSF samples. On average, 105 miRNAs were detected in each patient, equally distributed among $\mathrm{AD}$ patients and control patients with other types of dementia. We found no significant difference in total amount $(\mathrm{Ct})$ and number of different miRNAs in the two groups.

Fifty-two miRNAs were detected in CSF in at least nine out of ten patients in both groups. When comparing these 52 miRNAs between AD patients and controls, two miRNAs (let-7i-5p and miR-15a-5p) were found significantly up-regulated and one miRNA (miR-29c-3p) was found significantly down-regulated in patients with AD (Table 2, Fig. 2). However, when adjusting all $P$ values with the Benjamini-Hochberg procedure for multiple testing, none of them were statistically significant.

By combining two of the differentially expressed miRNAs (miR-29c-3p and miR-15a-5p) in a simple ratio model ( $R E L_{\text {miR-29c-3p }} / R L_{\text {miR-15a-5p }}$ ) AD patients could be separated from patients with other types of dementia (cutoff value 0.92 ) with a sensitivity of $90 \%$ and a specificity of $100 \%$ (Fig. 2 part D). To analyze the predictive power of each miRNA in order to categorize patients as having either $\mathrm{AD}$ or another type of dementia logistic regression analysis were done for the 52 frequently detected miRNAs in CSF (Fig. 3). The best predictors identified by this procedure were again miR-15a-5p and miR-29c-3p with classification accuracies of 0.8 and 0.7 , respectively.

When considering the detected miRNAs in CSF as categorical variables, three miRNAs were found to be expressed more often among $\mathrm{AD}$ patients compared to controls (Table 3). This difference was most significant for two miRNA in CSF (miR-199b-5p and miR-22-5p). Both miRNAs were detected in the CSF of $50 \%$ of AD patients and were not detected in the CSF of controls.

\section{miRNAs in blood}

A total of 308 different miRNAs were detected in the blood. Of these, 115 miRNAs were detected in all of the 20 blood samples. On average, 227 miRNAs were detected in each patient. One hundred and sixty-eight miRNAs were detected in at least nine out of 10 patients in each group (Additional file 1). Among these, miR$590-5 p$ (fold change $(\mathrm{FC})=1.35, p=0.002$ ) and miR-142$5 \mathrm{p}(\mathrm{FC}=1.22, p=0.043)$ were significantly up-regulated and miR-194-5p ( $\mathrm{FC}=0.54, p=0.028)$ was significantly down-regulated in $\mathrm{AD}$ patients compared to controls. Again, these $P$ values would not pass a BenjaminiHochberg correction for multiple testing.

When applying logistic regression analyses to blood data we identified 30 miRNAs with classification accuracies above 0.50 (i.e. random guessing), of which miR- 590-5p and miR-194-5p had the highest accuracy values (Additional file 2).

No candidates for categorical variables were identified in the blood.

\section{Blood-brain Barrier Function}

As a measure of BBB dysfunction, the albumin CSF/ serum concentration quotient $\left(\mathrm{Q}_{\mathrm{alb}}\right)$ is a widely accepted indicator [34]. Albumin is a serum protein that is normally prevented from passing into the CSF. However, under certain conditions such as neuroinflammation the BBB may become leaky allowing albumin molecules to pass from the blood. In this study, four out of ten AD patients had elevated $\mathrm{Q}_{\mathrm{alb}}$ values. Three patients in the control group, one with vascular dementia and two with frontotemporal dementia, also had elevated $\mathrm{Q}_{\mathrm{alb}}$ as sign of a leaky BBB (Additional file 3).

\section{Discussion}

Cognitive impairment may occur in a wide range of neurological, psychiatric and medical conditions, and the differential diagnosis of AD may be difficult, particularly against patients with other neurodegenerative or vascular brain diseases. Therefore we intended to set up a clinical relevant study design by choosing a non-healthy control group of patients with other types of dementia. Thus, with this exploratory investigation we aimed to identify expression differences of miRNAs in patients diagnosed with Alzheimer's disease and patients diagnosed with either vascular dementia, frontotemporal dementia or dementia with Lewy bodies in order to identify potential miRNA biomarker candidates with the ability to separate $\mathrm{AD}$ from other types of dementia. By carrying out a screening for 372 known miRNA sequences with RTqPCR we identified a differential expression pattern for let-7i-5p, miR-15a-5p, and miR-29c-3p in the CSF of AD patients and for miR-590-5p, miR-142-5p, and miR-194$5 \mathrm{p}$ in the blood of AD patients as compared to patients with other types of dementia.

\section{Overall miRNA detection}

On average, 105 different miRNAs were detected in the CSF of both cases and controls. These findings are consistent with results from other studies, including our own previous study of miRNA detection in CSF of stroke patients [35], in which a slightly smaller number of miRNAs were identified $(n=73)$ in each patient. The average number of detected miRNAs in blood was 227 as compared to 204 in our previous study, and the distribution patterns of miRNAs in blood and CSF in our studies have been very similar (Fig. 1) suggesting a reliable miRNA analysis. Data have been thoroughly checked using negative controls in the RT step and melting curve analysis. Furthermore, the steady levels of 
Table 2 Regulation of the 52 most frequently detected miRNAs in CSF of patients with dementia

\begin{tabular}{|c|c|c|c|c|c|c|}
\hline miRNA & $N_{A D}$ & $N_{\text {control }}$ & $M_{A D}$ (range) & $M_{\text {control }}$ (range) & $\mathrm{FC}$ & $p$ \\
\hline miR-15a-5p & 10 & 10 & $1.20(0.63-4.22)$ & $0.83(0.52-1.01)$ & 1.44 & 0.005 \\
\hline $\operatorname{miR}-29 c-3 p$ & 10 & 10 & $0.81(0.47-1.55)$ & $1.33(0.75-1.78)$ & 0.60 & 0.009 \\
\hline let-7i-5p & 10 & 10 & $1.31(0.67-3.23)$ & $0.85(0.27-1.27)$ & 1.54 & 0.019 \\
\hline miR-22-3p & 10 & 9 & $0.83(0.20-1.75)$ & $1.35(0.83-2.01)$ & 0.62 & n.s. \\
\hline miR-24-3p & 9 & 10 & $0.91(0.19-1.23)$ & $1.17(0.81-1.94)$ & 0.78 & n.s. \\
\hline miR-185-5p & 10 & 9 & $1.35(0.39-5.13)$ & $0.62(0.30-1.45)$ & 2.16 & n.s. \\
\hline miR-27b-3p & 10 & 10 & $0.95(0.49-1.48)$ & $1.21(0.63-1.87)$ & 0.78 & n.s. \\
\hline miR-142-3p & 9 & 9 & $1.19(0.27-4.31)$ & $0.86(0.26-1.83)$ & 1.38 & n.s. \\
\hline let-7 g-5p & 10 & 10 & $1.09(0.55-2.75)$ & $0.79(0.39-2.19)$ & 1.37 & n.s. \\
\hline miR-23a-3p & 10 & 10 & $0.92(0.53-1.55)$ & $1.11(0.62-2.04)$ & 0.82 & n.s. \\
\hline miR-23b-3p & 10 & 10 & $1.00(0.38-1.24)$ & $1.05(0.60-2.55)$ & 0.94 & n.s. \\
\hline miR-130a-3p & 10 & 10 & $0.82(0.41-1.56)$ & $1.22(0.42-2.13)$ & 0.67 & n.s. \\
\hline let-7c-5p & 10 & 10 & $0.88(0.37-2.21)$ & $1.15(0.35-3.09)$ & 0.76 & n.s. \\
\hline miR-150-5p & 10 & 9 & $0.89(0.23-5.44)$ & $0.82(0.37-1.68)$ & 1.09 & n.s. \\
\hline miR-361-5p & 9 & 9 & $0.95(0.47-1.32)$ & $1.00(0.70-1.51)$ & 0.94 & n.s. \\
\hline miR-25-3p & 10 & 9 & $1.00(0.36-5.49)$ & $0.87(0.34-1.77)$ & 1.14 & n.s. \\
\hline miR-221-3p & 10 & 10 & $1.68(0.15-3.04)$ & $1.22(0.15-1.70)$ & 1.38 & n.s. \\
\hline miR-29a-3p & 10 & 10 & $0.96(0.72-1.30)$ & $1.01(0.71-1.71)$ & 0.94 & n.s. \\
\hline miR-30a-5p & 10 & 10 & $1.32(0.20-2.91)$ & $1.12(0.37-1.91)$ & 1.17 & n.s. \\
\hline miR-10b-5p & 9 & 10 & $0.90(0.26-5.08)$ & $1.04(0.21-1.70)$ & 0.86 & n.s. \\
\hline let-7a-5p & 10 & 10 & $0.80(0.29-2.58)$ & $1.14(0.65-3.49)$ & 0.70 & n.s. \\
\hline miR-125b-5p & 10 & 10 & $0.85(0.62-1.53)$ & $0.98(0.69-2.77)$ & 0.87 & n.s. \\
\hline miR-16-5p & 10 & 10 & $0.96(0.05-9.45)$ & $0.98(0.56-1.47)$ & 0.98 & n.s. \\
\hline miR-424-5p & 10 & 10 & $1.19(0.35-3.38)$ & $0.72(0.47-2.38)$ & 1.65 & n.s. \\
\hline miR-143-3p & 10 & 10 & $1.04(0.45-1.48)$ & $0.92(0.69-2.32)$ & 1.12 & n.s. \\
\hline miR-19a-3p & 9 & 10 & $1.14(0.21-3.68)$ & $1.15(0.65-1.49)$ & 0.99 & n.s. \\
\hline miR-124-3p & 10 & 9 & $1.31(0.26-2.73)$ & $1.21(0.32-2.08)$ & 1.08 & n.s. \\
\hline let-7b-5p & 10 & 10 & $1.03(0.31-3.50)$ & $0.83(0.45-3.39)$ & 1.23 & n.s. \\
\hline miR-146a-5p & 9 & 10 & $1.37(0.23-2.40)$ & $0.91(0.20-2.69)$ & 1.50 & n.s. \\
\hline let-7d-3p & 9 & 10 & $1.02(0.54-1.89)$ & $1.33(0.62-1.83)$ & 0.76 & n.s. \\
\hline miR-125a-5p & 10 & 9 & $1.21(0.32-2.96)$ & $0.71(0.29-2.68)$ & 1.69 & n.s. \\
\hline miR-27a-3p & 9 & 10 & $1.14(0.15-2.81)$ & $1.29(0.25-3.03)$ & 0.88 & n.s. \\
\hline miR-30d-5p & 10 & 10 & $1.16(0.31-2.47)$ & $1.15(0.34-1.83)$ & 1.01 & n.s. \\
\hline miR-99a-5p & 10 & 10 & $0.98(0.57-1.36)$ & $1.07(0.62-1.93)$ & 0.91 & n.s. \\
\hline miR-145-5p & 10 & 10 & $1.16(0.30-1.73)$ & $1.00(0.47-2.05)$ & 1.15 & n.s. \\
\hline miR-100-5p & 10 & 10 & $0.92(0.54-1.88)$ & $1.07(0.63-1.86)$ & 0.85 & n.s. \\
\hline miR-223-3p & 9 & 10 & $1.14(0.86-2.74)$ & $0.78(0.22-4.81)$ & 1.45 & n.s. \\
\hline miR-320a & 10 & 10 & $0.94(0.54-1.62)$ & $1.05(0.64-1.68)$ & 0.90 & n.s. \\
\hline miR-497-5p & 10 & 10 & $1.07(0.51-1.72)$ & $1.08(0.64-1.81)$ & 0.98 & n.s. \\
\hline miR-373-5p & 9 & 10 & $1.10(0.26-2.66)$ & $1.11(0.30-3.44)$ & 0.98 & n.s. \\
\hline miR-204-5p & 10 & 10 & $1.05(0.07-2.30)$ & $0.99(0.47-2.28)$ & 1.06 & n.s. \\
\hline miR-92a-3p & 10 & 10 & $0.94(0.70-3.69)$ & $0.84(0.47-2.17)$ & 1.12 & n.s. \\
\hline miR-423-5p & 10 & 10 & $1.11(0.65-2.27)$ & $0.78(0.44-2.22)$ & 1.42 & n.s. \\
\hline miR-19b-3p & 10 & 10 & $1.08(0.18-2.25)$ & $1.20(0.21-2.20)$ & 0.90 & n.s. \\
\hline
\end{tabular}


Table 2 Regulation of the 52 most frequently detected miRNAs in CSF of patients with dementia (Continued)

\begin{tabular}{lllllll}
\hline miR-9-5p & 9 & 10 & $1.25(0.50-1.97)$ & $1.24(0.24-3.11)$ & 1.00 & n.s. \\
miR-30c-5p & 10 & 10 & $1.15(0.20-2.57)$ & $0.99(0.37-2.38)$ & 1.15 & n.s. \\
miR-21-5p & 10 & 10 & $0.89(0.66-2.29)$ & $1.06(0.27-1.86)$ & 0.83 & n.s. \\
miR-34a-5p & 10 & 9 & $0.99(0.26-2.39)$ & $1.06(0.46-2.07)$ & 0.94 & n.s. \\
miR-101-3p & 10 & 9 & $1.11(0.37-1.48)$ & $0.93(0.56-2.64)$ & 1.19 & n.s. \\
miR-29b-3p & 9 & 9 & $1.12(0.31-1.76)$ & $0.92(0.49-2.02)$ & 1.22 & n.s. \\
miR-199a-3p & 10 & 10 & $0.92(0.58-3.40)$ & $1.16(0.39-1.67)$ & 0.79 & n.s. \\
miR-9-3p & 10 & 10 & $1.13(0.23-2.35)$ & $1.02(0.28-2.86)$ & 1.09 & n.s. \\
\hline
\end{tabular}

$N_{A D}$ number of Alzheimer patients in which the miRNA was detected, $N_{\text {control }}$ number of control patients in which the miRNA was detected, $M_{A D}$ the median of relative expression levels among Alzheimer patients, $M_{\text {control }}$ median of relative expression levels among control patients, $F C$ fold change of median values, $p$ p-values of difference calculated by two-sided nonparametric Mann-Whitney test (not corrected for multiple testing), n.s. nonsignificant ( $p>0.05$ )

DNA and RNA spike-ins indicate good technical performance of our profiling experiment.

\section{Findings in CSF}

In the CSF we identified a differential expression pattern for miR-15a-5p, let-7i-5p, and miR-29c-3p. In our study, miR-15a-5p was up-regulated with a fold change of 1.44 $(p=0.005)$ in the CSF of AD patients. Bekris et al. [13] have previously found elevated levels of miR-15a in plasma and hippocampal tissue of $\mathrm{AD}$ patients with a positive correlation to plaque scores. By contrast, Hebert et al. [15] have found miR-15a down-regulated in cerebral cortex of $\mathrm{AD}$ patients compared to healthy controls. Interestingly, miR-15a's regulation of both APP and BACE1 has been validated in various ways, including reporter assay analysis $[15,36]$. Since the miRNAmRNA binding usually results in gene silencing via translational repression, one could argue that the level of miR-15a-5p was expected to be lower in AD patients compared to other types of dementia. However, as the complex pathogenesis of late-onset $\mathrm{AD}$ is not limited to an increased $A \beta$ production but also involves impaired $A \beta$ clearance [37], the possible ways for interaction of miR-15a-5p in AD biology are many. Among the list of

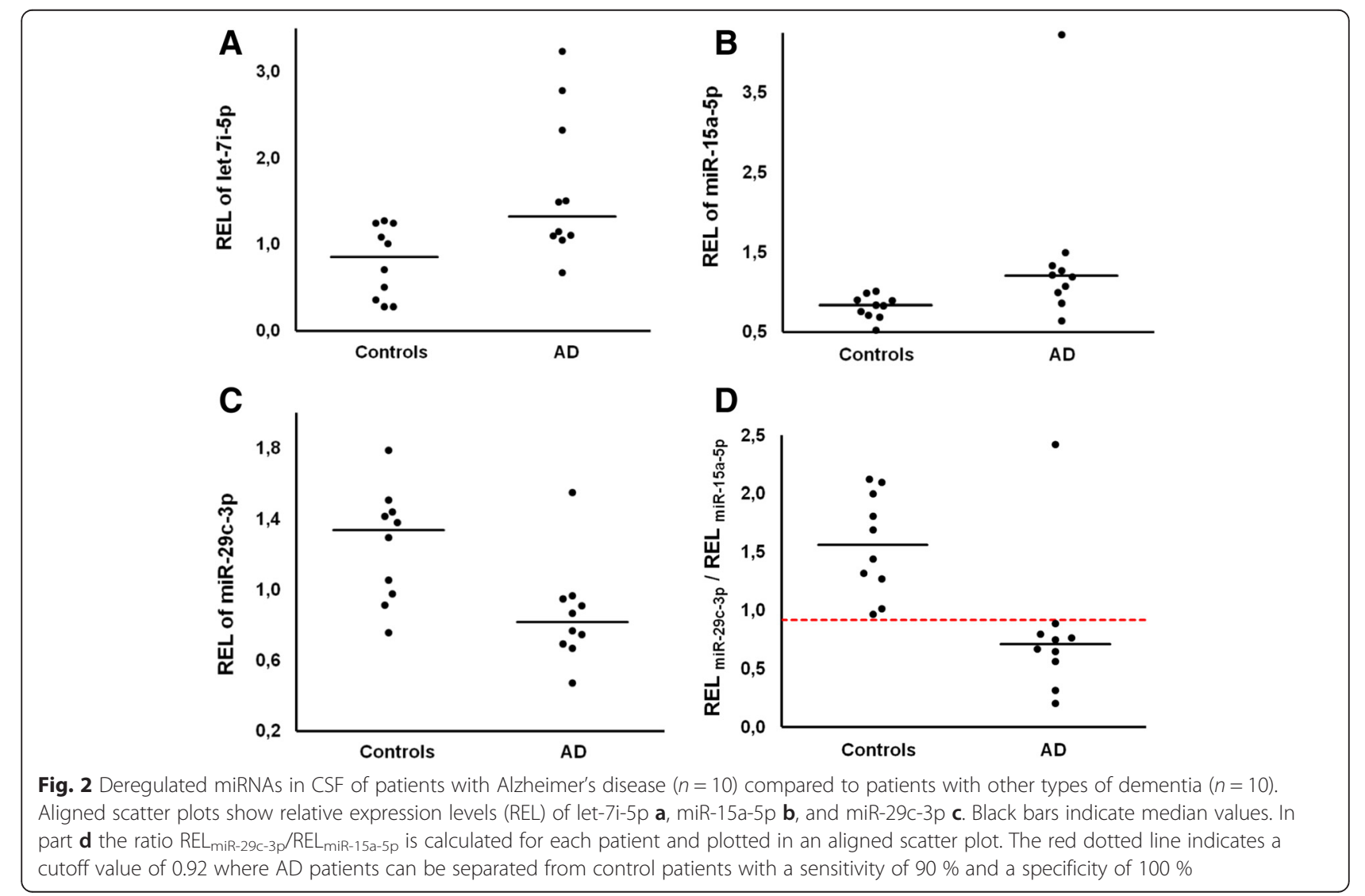




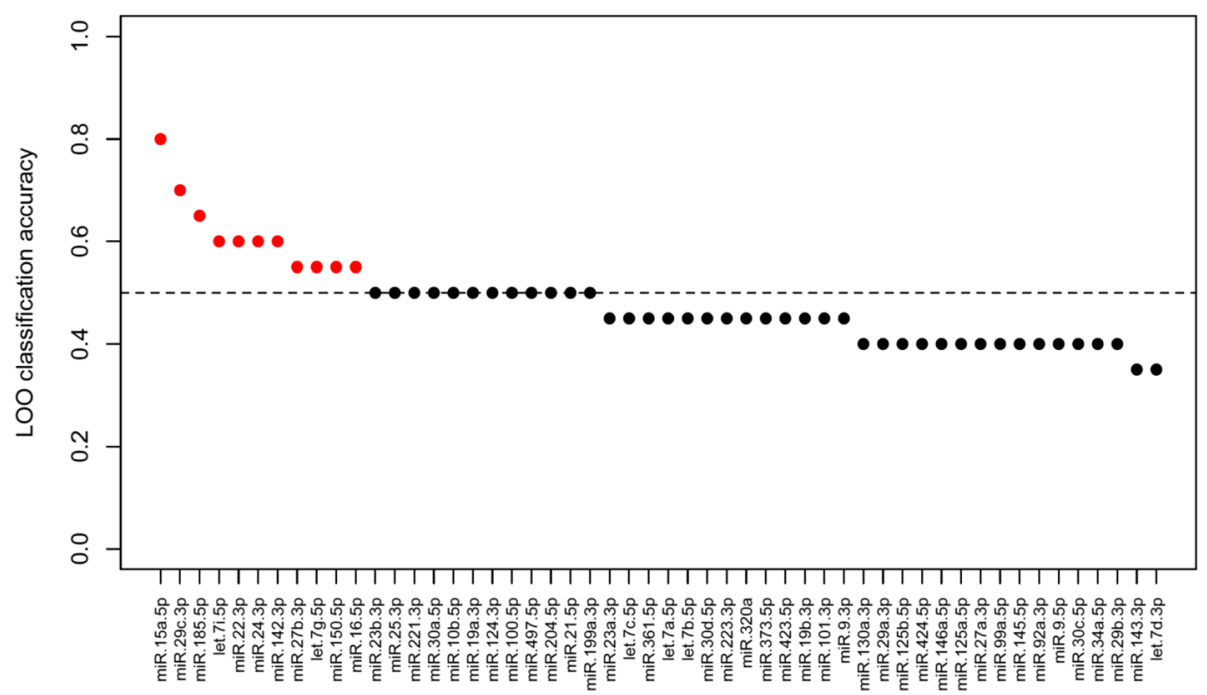

Fig. 3 Logistic regression cross-validated classification accuracies based on a leave-one-out procedure (LOO). The 52 most frequently detected miRNAs in CSF are ordered by decreasing magnitude of predictive power. Red color indicates predictors with classification accuracy above random guessing

predicted targets for miR-15a obtained from the miRWalk database [38] we discovered other genes relevant to late-onset $\mathrm{AD}$ [39] including CD2-associated protein (CD2AP) which recently has been suggested to mediate the integrity of the BBB [40].

In the CSF of $\mathrm{AD}$ patients we also found an upregulation of let-7i-5p $(\mathrm{FC}=1.54, p=0.019)$ compared to other types of dementia. Burgos et al. [26] reported a positive correlation between the level of let-7i-3p in serum of AD patients and their Braak stages. Even more interesting, Lehmann et al. [20] found let-7b, another member of the let-7 family of miRNAs, elevated in the CSF of AD patients. Through reporter assay experiments and by intrathecal injection of let-7b into the CSF of wild-type mice Lehmann et al. showed that extracellular let-7b activated the RNA-sensning Toll-like receptor 7 (TLR7) in both immune cells and neurons, and that this activation resulted in neuronal cell death. From these experiments the authors hypothesized that extracellular miRNAs like let-7 can contribute to spread neuronal damage from one brain region through activation of extracellular TLR4. Also Let-7i-5p, which was elevated

Table 3 miRNAs detected more often in CSF samples of AD patients compared to patients with other types of dementia

\begin{tabular}{lllllll}
\hline miRNA & $N_{A D}$ & $N_{\text {control }}$ & OR & Sensitivity & Specificity & $p$ \\
\hline miR-199b-5p & $5 / 10$ & $0 / 10$ & 21 & 50 & 100 & $0.03^{*}$ \\
miR-22-5p & $5 / 10$ & $0 / 10$ & 21 & 50 & 100 & $0.03^{*}$ \\
miR-206 & $6 / 10$ & $1 / 10$ & 13.5 & 60 & 90 & 0.06
\end{tabular}

$N_{A D}$ number of patients with Alzheimer's disease in which the miRNA was detected, $N_{\text {control }}$ number of patients with other types of dementia in which the miRNA was detected, OR odds ratio, $p$ p-value (Fischer's exact test). Star indicates statistically significance $(p<0.05)$ in the CSF of AD patients in our study, has specifically been validated to target TLR7 [41].

We found miR-29c-3p down-regulated in the CSF of $\mathrm{AD}$ patients with a FC of $0.60(p=0.009)$. This finding is in accordance with the results from Hebert et al. [15] as previously mentioned, who found a significant downregulation of miR-29a and miR-29b (other members of the miR-29 family) in temporal and cerebellar cortical tissue from sporadic AD patients compared to healthy controls. BACE1 was validated as a target of miR-29a/b by reporter assays, and furthermore the inhibiting effect of miR-29a/b on BACE1 activity was corroborated by gain- and loss of function experiments using HEK293 cells. In mice brain tissue and cell cultures, the suppressing effect of miR-29c-3p (down-regulated in our study) on BACE1 has also been validated by Zong et al. [42] by various methods. Most recently, Lei et al. [11] found down-regulation of miR-29c in brain tissues from sporadic AD patients in whom the miR-29c level was negatively correlated with BACE1 mRNA level. In contrast to these experiments and to our results (Table 2), Kiko et al. [25] have found significant up-regulation of miR-29a and miR-29b in the CSF of AD patients compared to healthy control subjects. In this context, it has, however, not been clarified whether each member of the miR-29 family of miRNAs is expected to be regulated in the same direction.

When considering the detected miRNAs in CSF as categorical variables, three miRNAs were found to be expressed more often in the CSF of AD patients compared to controls (Table 3), namely miR-199b-5p, miR22-5p, and miR-206. Taking into account the small study population and the large number of observations in our 
qPCR experiment, however we cannot exclude the possibility of randomness in this observation. The expression of miR-206 has been linked to the regulation of brainderived neurotrophic factor (BDNF), a regulator of synaptic plasticity and memory, which is known to be deficient in $\mathrm{AD}$ brains. Lee et al. [43] found up-regulation of miR-206 in human temporal cortex of AD patients and in transgenic $\mathrm{AD}$ mice, and validated its repression of BDNF by northern blotting and qPCR. Similarly, Tian et al. [44] reported an up-regulation of miR-206 in hippocampal tissue, cerebrospinal fluid, and plasma of embryonic APP/PS1 transgenic mice, and found that miR-206 depressed the expression of BDNF.

To our knowledge, the possible functions miR-199b-5p and miR-22-5p in relation to $\mathrm{AD}$ and other dementias are unknown.

\section{Findings in blood}

Despite the large number of frequently detected miRNAs in the blood $(n=168)$, only three miRNAs (miR-590-5p, miR-142-5p, and miR-194-5p) were identified as being differential expressed between AD patients and controls.

The expression of miR-590-5p, which was up-regulated in the blood of $\mathrm{AD}$ patients in our study, has previously been reported up-regulated in plasma of patients with vulnerable coronary artery disease [45]. The antisense string, miR-590-3p, has been investigated in an extensive study involving 274 patients with frontotemporal dementia, 287 patients with $\mathrm{AD}$, and 344 non-demented agematched controls [46]. In this study the expression of miR590-3p was decreased in mononuclear cells in peripheral blood of AD patients compared to non-demented control subjects, and its target hnRNPA1, a protein involved in the maturation of APP, was up-regulated. The study is not directly comparable to our experiment mainly because of the different cells and body fluids examined, and therefore the meaning of the oppositely directed regulation of miR590 found in these two studies remains unclear.

With regard to miR-142-5p, which was up-regulated in the blood of AD patients in our study, the only validated target is nuclear factor, erythroid 2-like 2 (NRF2). This transcription factor regulates several genes that encode proteins involved in responses to injury and inflammation which includes the production of free radicals [47]. Results from other clinical studies are in some ways different from ours. Cogswell et al. [18] found miR142-5p down-regulated in the CSF of AD patients compared to controls, and Kumar et al. [48] found the antisense string, miR-142-3p, down-regulated in plasma of $\mathrm{AD}$ patients compared to both MCI patients and healthy controls.

In our study, miR-194-5p was down-regulated in the blood of AD patients. We have no knowledge from previous studies or from literature searches about the role and function of this miRNA in relation to $\mathrm{AD}$ or other types of dementia.

Another miRNA, miR-107, which we found nonsignificantly down-regulated in the blood of AD patients, but which had a relatively high classification accuracy (Additional file 2), has interestingly been reported downregulated in the blood of $\mathrm{AD}$ patients in two previous studies $[49,50]$. This miRNA is known to target BACE1, and the level of miR-107 was previously found downregulated in $\mathrm{AD}$ brain tissue, suggesting a role for miR107 in the pathogenesis of AD [10].

In summary, most of the deregulated miRNAs that we have identified in both CSF and blood can be linked to relevant target molecules, although the overall picture of our findings is still unclear and warrants further elucidation. Existing studies differ in design and extraction methods making it difficult to compare results and to gain a complete overview of miRNAs affected by AD.

\section{Best biomarker candidates}

A biomarker measured in the blood is preferable to more invasive alternatives. Blood samples from patients are obtained easily at a low cost and at a low risk of adverse effects. However, blood is a systemic fluid which composition may reflect processes in other tissues or organs besides the brain. CSF, on the other hand, which is protected by the blood-brain barrier, ought to be a reliable biomarker source for neurodegenerative diseases in the central nervous system. In our study, the results from miRNA expression analysis in the CSF seem more interesting compared to blood data in various ways. Firstly, an equal number of miRNAs were differentially expressed in blood and CSF despite the four times larger number of frequently detected miRNAs in the blood. Secondly, we find it interesting that the three differential expressed miRNAs in CSF (miR-29c$3 p$, miR15a-5p, and let-7i-5p) could all be related to AD relevant targets like APP and BACE1.

miR-29c-3p and miR-15a-5p had the highest classification accuracies separately, and by combing them in a simple ratio model $\left(\mathrm{REL}_{\mathrm{miR}-29 \mathrm{c}-3 \mathrm{p}} / \mathrm{REL}_{\mathrm{miR}-15 \mathrm{a}-5 \mathrm{p}}\right) \mathrm{AD}$ patients could be separated from patients with other types of dementia (cutoff value 0.92 ) with a sensitivity of $90 \%$ and a specificity of $100 \%$. This ratio model is of course associated with statistical uncertainty due to our small population size, and should therefore only be seen as a proposal for the use of these markers in future studies, which are highly needed. Logistic regression models that combines more than one miRNA is another approach, but again in this small sized pilot study it is impossible to select a single best model according to the low stability proportion of each predictor and none of our $P$ values were strong enough to be considered significant after correction for multiple testing. 


\section{The impact of the BBB}

Although the CSF is isolated from the rest of the circulation by the blood-brain barrier, under certain conditions, such as inflammation, the barrier can become leaky allowing molecules to pass from the blood. To measure the BBB function we calculated CSF/serum albumin ratios $\left(\mathrm{Q}_{\mathrm{alb}}\right)$ in the 20 patients included. In this study, four out of ten $A D$ patients had elevated $\mathrm{Q}_{\text {alb }}$ values. Three patients in the control group also had elevated $\mathrm{Q}_{\mathrm{alb}}$ values indicating a leaky BBB. Thus, in those patients the presence of miRNAs in the CSF could simply be a result of contamination from the systemic circulation. When examining the expression of miR-225p, miR-199b-5p and miR-206 (detected in the CSF with a frequency of $50 \%$ in the $\mathrm{AD}$ group and $0 \%$ in the control group) we found no association between the expression of these miRNAs in the blood and CSF of patients with an elevated $\mathrm{Q}_{\mathrm{alb}}$, suggesting that their presence in CSF does not seem to be explained by a leak in BBB. Also, we did not find an obvious association between the expression of other miRNAs in blood and CSF, although a direct correlation analysis between the levels in blood and CSF was prevented due to the small size of our study population. Lastly we noticed that some miRNAs which have been characterized as brain specific, such as miR-124 and miR-9, were detected predominantly in the CSF, further indicating that the expression of miRNAs in the two body fluids is individually regulated and probably unaffected by small disturbances in the BBB function. Findings by Bekris et al. [13] and Burgos et al. [26] support this notion since they also did not find any correlation between the expression of individual miRNAs in CSF and blood in their studies. Neither of these two studies included other specific measures to evaluate the integrity of the $\mathrm{BBB}$ whereas we calculated the $\mathrm{Q}_{\mathrm{alb}}$ as a measure of BBB dysfunction [34]. The findings taken together should, however, be interpreted with caution and further studies are warranted to clarify the origin of miRNAs in the CSF and their transport across the BBB.

\section{Conclusions}

Detection of miRNA expression profiles in blood and in particular CSF of patients diagnosed with different types of dementia is feasible and it seems that several expressional differences between $\mathrm{AD}$ and other dementia types do exist in a clinically relevant setup like the present. In this explorative pilot study, the deregulated miRNAs in CSF of AD patients compared to a relevant non-healthy control group may be associated with target genes related to AD pathology, including APP and BACE1, which suggests that miRNAs are interesting candidates for $\mathrm{AD}$ biomarkers in the future.

Clinical validation of our findings in a larger scale is obviously needed. We are currently working on a validation study with larger study groups that will enable subgroup analyses between the different types of dementia and also allow us to make stronger model assumptions based on combinations of several miRNAs as diagnostic biomarkers of the different types of dementia.

\section{Ethics approval and consent to participate}

A statement on ethics approval and consent to participate is included in the methods section.

\section{Consent for publication}

All included patients gave written and oral informed consent for publication.

\section{Availability of data and materials}

The datasets supporting the conclusions of this article are included within the article and its additional files.

\section{Additional files}

Additional file 1: Regulation of the 168 most frequently detected miRNAs in blood of patients with dementia. $N_{A D}$ number of Alzheimer patients in which the miRNA was detected, $N_{\text {control }}$ number of control patients in which the miRNA was detected, $M_{A D}$ the median of relative expression levels among Alzheimer patients, $M_{\text {control }}$ median of relative expression levels among control patients, FC fold change of median values, $p$ p-values of difference calculated by two-sided nonparametric Mann-Whitney test (not corrected for multiple testing), n.s. nonsignificant ( $p>0.05)$. (PDF $412 \mathrm{~kb})$

Additional file 2: Logistic regression cross-validated classification accuracies based on a leave-one-out procedure (LOO). The 168 most frequently detected miRNAs in blood are ordered by decreasing magnitude of predictive power. Red color indicates predictors with classification accuracy above random guessing. (PDF $146 \mathrm{~kb}$ )

Additional file 3: Estimation of the blood brain barrier dysfunction in patients with dementia based on albumin CSF/serum concentration quotients. AD Alzheimer's disease, DLB dementia with Lewy bodies, VAD vascular dementia, FTD frontotemporal dementia. Qalb albumin CSF/ serum concentration quotient. Qalb limit age dependent upper limit of albumin CSF/serum concentration quotient calculated by the formula $Q_{\text {alb }}=\left(4+\frac{\text { age }}{15}\right)$. BBB blood brain barrier. (PDF $\left.447 \mathrm{~kb}\right)$

\section{Abbreviations \\ miRNAs: MicroRNAs; CSF: cerebrospinal fluid; AD: Alzheimer's disease; AB1-42: amyloid beta; mRNA: messenger RNA; BACE1: amyloid precursor protein cleaving enzyme 1; BBB: blood-brain barrier; PD: Parkinson's disease; FTD: frontotemporal dementia; DLB: dementia with Lewy bodies; VAD: vascular dementia; MMSE: Mini-Mental State Examination; \\ ACE: Addenbrooke's Cognitive Examination; CT: Computer Tomography; MRI: Magnetic Resonance Imaging; F-FDG-PET: Fluorodeoxyglucose Positron Emission Tomography; DAT: Dopamine Active Transporter; qPCR: quantitative polymerase chain reactions; REL: relative expression level; LOO: leave-one-out cross-validation procedure; FC: fold change; Qalb: albumin CSF/serum concentration quotient.}

\section{Competing interests}

The authors declare that they have no competing interests.

\section{Authors' contributions}

Sørensen SS participated in the design of the study and its coordination, included the patients, collected CSF and blood samples, handled CSF and blood samples prior to miRNA analysis, performed data normalization and statistical analysis, and drafted the manuscript. Nygaard A-B participated in 
the design of the study, helped to handle CSF and blood samples prior to miRNA analyses, helped with the statistical analysis, and helped to draft the manuscript. Christensen TC conceived the study, participated in its design and coordination, participated in the statistical analysis, and helped to draft the manuscript. All authors read and approved the final manuscript.

\section{Authors information}

Sofie Sølvsten Sørensen holds the title M.D. and is currently enrolled as Ph.D. student at the University of Copenhagen. Ann-Britt Nyggard holds the titles M.Sc, and Ph.D. Thomas Christensen is a specialist in neurology and holds the title D.M.Sc.

\section{Acknowledgements}

We greatly thank all personnel at the Department of Neurology, Copenhagen University Hospital, Hillerød, who helped us handling the study patients, including the neuropsychologists (especially Anne Margrethe Nissen), the nurses and health care assistants (especially Anne-Mette Larsen), and the doctors at the Memory Clinic. Thank you to Andreas Kryger Jensen, Department of Biostatistics, University of Copenhagen, who provided statistical assistance with logistic regression analyses and contributed with Fig. 3 and Additional file 2. We also thank the chief consultant at the Department of Neurology, Kai Jensen, who provided all facilities and helped to organize the study.

\section{Funding}

This project was supported by grants from the Research Foundation of Nordsjællands Hospital, the Research Foundation for Health Research of the Capital Region of Denmark, and Helen Rude's Foundation.

\section{Author details}

'Department of Neurology, Copenhagen University Hospital, Nordsjællands Hospital, Dyrehavevej 29, 3400 Hillerød, Denmark. ${ }^{2}$ Department of Clinical Biochemistry, Copenhagen University Hospital, Nordsjællands Hospital, Dyrehavevej 29, 3400 Hillerød, Denmark.

\section{Received: 15 December 2015 Accepted: 28 February 2016 Published online: 15 March 2016}

\section{References}

1. Qiu C, Fratiglioni L. Epidemiology of Alzheimer's disease. In: Waldemar G, Burns A, editors. Alzheimer's Disease. Oxford: Oxford Neuropsyciatric Library; 2009. p. 17-27.

2. Prince $M$, Bryce $R$, Albanese $E$, Wimo A, Ribeiro W, Ferri CP. The global prevalence of dementia: a systematic review and metaanalysis. Alzheimers Dement. 2013;9:63-75

3. Wimo A, Jonsson L, Winblad B. An estimate of the worldwide prevalence and direct costs of dementia in 2003. Dement Geriatr Cogn Disord. 2006;21:175-81.

4. LaFerla FM, Green KN, Oddo S. Intracellular amyloid-beta in Alzheimer's disease. Nat Rev Neurosci. 2007;8:499-509.

5. Ballatore C, Lee VM-Y, Trojanowski JQ. Tau-mediated neurodegeneration in Alzheimer's disease and related disorders. Nat Rev Neurosci. 2007:8:663-72.

6. Aliev G, Palacios HH, Walrafen B, Lipsitt AE, Obrenovich ME, Morales L. Brain mitochondria as a primary target in the development of treatment strategies for Alzheimer disease. Int J Biochem Cell Biol. 2009;41:1989-2004.

7. Heneka MT, Carson MJ, El Khoury J, Landreth GE, Brosseron F, Feinstein DL, Jacobs AH, Wyss-Coray T, Vitorica J, Ransohoff RM, Herrup K, Frautschy S a, Finsen B, Brown GC, Verkhratsky A, Yamanaka K, Koistinaho J, Latz E, Halle A, Petzold GC, Town T, Morgan D, Shinohara ML, Perry VH, Holmes C, Bazan NG, Brooks DJ, Hunot S, Joseph B, Deigendesch N. Neuroinflammation in Alzheimer's disease. Lancet Neurol. 2015;14:388-405.

8. Rosa Ml, Perucchi J, Medeiros LR, et al. Accuracy of cerebrospinal fluid $A \beta(1-42)$ for Alzheimer's disease diagnosis: a systematatic review and metaanalysis. J Alzheimers Dis. 2014;40:443-54.

9. Van Harten AC, Kester MI, Visser PJ, Blankenstein MA, Pijnenburg YAL, Van Der Flier WM, Scheltens P. Tau and p-tau as CSF biomarkers in dementia: A meta-analysis. Clin Chem Lab Med. 2011;49:353-66.

10. Wang W-X, Rajeev BW, Stromberg AJ, Ren N, Tang G, Huang Q, Rigoutsos I, Nelson PT. The expression of microRNA miR-107 decreases early in Alzheimer's disease and may accelerate disease progression through regulation of beta-site amyloid precursor protein-cleaving enzyme 1. J Neurosci. 2008;28:1213-23.
11. Lei $X$, Lei L, Zhang Z, Zhang Z, Cheng Y. Downregulated miR-29c correlates with increased BACE1 expression in sporadic Alzheimer' s disease. Int J Clin Exp Pathol. 2015;8:1565-74.

12. Nelson PT, Wang W-X. MiR-107 is reduced in Alzheimer's disease brain neocortex: validation study. J Alzheimers Dis. 2010;21:75-9.

13. Bekris LM, Lutz F, Montine TJ, Yu CE, Tsuang D, Peskind ER, Leverenz JB. MicroRNA in Alzheimer's disease: an exploratory study in brain, cerebrospinal fluid and plasma. Biomarkers. 2013;18:455-66.

14. Wang W-X, Huang Q, Hu Y, Stromberg AJ, Nelson PT. Patterns of microRNA expression in normal and early Alzheimer's disease human temporal cortex: white matter versus gray matter. Acta Neuropathol. 2011;121:193-205.

15. Hébert SS, Horré K, Nicolaï L, Papadopoulou AS, Mandemakers W, Silahtaroglu AN, Kauppinen S, Delacourte A, De Strooper B. Loss of microRNA cluster miR-29a/b-1 in sporadic Alzheimer's disease correlates with increased BACE1/beta-secretase expression. Proc Natl Acad Sci U S A. 2008;105:6415-20.

16. Lukiw WJ. Micro-RNA speciation in fetal, adult and Alzheimer's disease hippocampus. Neuroreport. 2007;18:297-300.

17. Sethi P, Lukiw WJ. Micro-RNA abundance and stability in human brain: specific alterations in Alzheimer's disease temporal lobe neocortex. Neurosci Lett. 2009;459:100-4.

18. Cogswell JP, Ward J, Taylor IA, Waters M, Shi Y, Cannon B, Kelnar K, Kemppainen J, Brown D, Chen C, Prinjha RK, Richardson JC. Identification of miRNA Changes in Alzheimer's Disease Brain and CSF Yields Putative Biomarkers and Insights into Disease Pathways. J Alzheimer's Dis. 2008;14:27-41.

19. Coolen M, Katz S, Bally-Cuif L. miR-9: a versatile regulator of neurogenesis. Front Cell Neurosci. 2013;7(November):220.

20. Lehmann SM, Krüger C, Park B, Derkow K, Rosenberger K, Baumgart J, Trimbuch T, Eom G, Hinz M, Kaul D, Habbel P, Kälin R, Franzoni E, Rybak A, Nguyen D, Veh R, Ninnemann O, Peters O, Nitsch R, Heppner FL, Golenbock D, Schott E, Ploegh HL, Wulczyn FG, Lehnardt S. An unconventional role for miRNA: let-7 activates Toll-like receptor 7 and causes neurodegeneration. Nat Neurosci. 2012;15:827-35.

21. Alexandrov PN, Dua P, Hill JM, Bhattacharjee $S$, Zhao Y, Walter J. microRNA (miRNA) speciation in Alzheimer' s disease (AD) cerebrospinal fluid (CSF) and extracellular fluid (ECF). Int J Biochem Mol Biol. 2012;3:365-73.

22. Müller M, Kuiperij HB, Claassen JA, Küsters B, Verbeek MM. MicroRNAs in Alzheimer's disease: differential expression in hippocampus and cell-free cerebrospinal fluid. Neurobiol Aging. 2014;35:152-8.

23. Sala Frigerio C, Lau P, Salta E, Tournoy J, Bossers K, Vandenberghe R, Wallin A, Bjerke M, Zetterberg H, Blennow K, De Strooper B. Reduced expression of hsamiR-27a-3p in CSF of patients with Alzheimer disease. Neurology. 2013;81:2103-6.

24. Liu C-G, Wang J-L, Li L, Xue L-X, Zhang Y-Q, Wang P-C. MicroRNA-135a and $-200 \mathrm{~b}$, potential Biomarkers for Alzheimer $s$ disease, regulate $\beta$ secretase and amyloid precursor protein. Brain Res. 2014;1583:55-64.

25. Kiko T, Nakagawa K, Tsuduki T, Furukawa K, Arai H, Miyazawa T. MicroRNAs in plasma and cerebrospinal fluid as potential markers for Alzheimer's disease. J Alzheimer's Dis. 2014;39:253-9.

26. Burgos K, Malenica I, Metpally R, Courtright A, Rakela B, Beach T, Shill H, Adler C, Sabbagh M, Villa S, Tembe W, Craig D, Van Keuren-Jensen K. Profiles of extracellular miRNA in cerebrospinal fluid and serum from patients with Alzheimer's and Parkinson's diseases correlate with disease status and features of pathology. PLoS One. 2014;9:e94839.

27. Galimberti D, Villa C, Fenoglio C, Serpente M, Ghezzi L, Cioffi SMG, Arighi A, Fumagalli G, Scarpini E. Circulating miRNAs as Potential Biomarkers in Alzheimer's Disease. J Alzheimers Dis. 2014;42:1261-7.

28. McKhann G, Knopman DS, Chertkow H, Hymann B, Jack CR, Kawas C, Klunk W, Koroshetz W, Manly J, Mayeux R, Mohs R, Morris J, Rossor M, Scheltens P, Carrillo M, Weintrub S, Phelphs C. The diagnosis of dementia due to Alzheimer's disease: Recommendations from the National Institute on Aging- Alzheimer's Association workgroups on diagnostic guidelines for Alzheimer's disease. Alzheimers Dement. 2011;7:263-9.

29. Roman G. Vascular dementia: diagnostic criteria for research studies. Report of the NINDS-AIREN International Workshop. Neurology. 1994:43:250-60.

30. McKhann GM. Clinical and Pathological Diagnosis of Frontotemporal Dementia. Arch Neurol. 2001:58:1803.

31. McKeith IG, Galasko D, Kosaka K, Perry EK, Dickson DW, Hansen LA, Salmon DP, Lowe J, Mirra SS, Byrne EJ, Lennox G, Quinn NP, Edwardson J a, Ince PG, Bergeron C, Burns a, Miller BL,Lovestone S, Collerton D, Jansen EN, Ballard C, 
de Vos R a, Wilcock GK, Jellinger K a, Perry RH. Consensus guidelines for the clinical and pathologic diagnosis of dementia with Lewy bodies (DLB): report of the consortium on DLB international workshop. Neurology. 1996;47:1113-24.

32. Andersen $C L$, Jensen $J L$, Ørntoft TF. Normalization of Real-Time Quantitative Reverse Transcription-PCR Data : A Model-Based Variance Estimation Approach to Identify Genes Suited for Normalization, Applied to Bladder and Colon Cancer Data Sets Normalization of RealTime Quantitative Reverse. Cancer Res. 2004;64:5245-50.

33. Jolla L. Quantification strategies in real-time PCR Michael W.Pfaffl. 2004. p. 87-112.

34. Reiber H, Peter JB. Cerebrospinal fluid analysis: disease-related data patterns and evaluation programs. J Neurol Sci. 2001;184:101-22.

35. Sørensen SS, Nygaard A-B, Nielsen M-Y, Jensen K, Christensen T. miRNA Expression Profiles in Cerebrospinal Fluid and Blood of Patients with Acute Ischemic Stroke. Transl Stroke Res. 2014;5:711-8.

36. Hébert SS, Horré K, Nicolaï L, Bergmans B, Papadopoulou AS, Delacourte A De Strooper B. MicroRNA regulation of Alzheimer's Amyloid precursor protein expression. Neurobiol Dis. 2009;33:422-8.

37. Mawuenyega KG, Sigurdson W, Ovod V, Munsell L, Kasten T, Morris JC, Yarasheski KE, Bateman RJ. Decreased clearance of CNS beta-amyloid in Alzheimer's disease. Science. 2010;330(6012):1774.

38. Dweep H, Gretz N. miRWalk2.0: a comprehensive atlas of microRNA-target interactions. Nat Methods. 2015;12:697.

39. Calero M, Gomez-Ramos A, Calero O, Soriano E, Avila J, Medina M. Additional mechanisms conferring genetic susceptibility to Alzheimer's disease. Front Cell Neurosci. 2015;9:1-9.

40. Cochran JN, Rush T, Buckingham SC, Roberson ED. The Alzheimer's disease risk factor CD2AP maintains blood-brain barrier integrity. Hum Mol Genet. 2015;24:6667-74.

41. Chen XM, Splinter PL, O'Hara SP, LaRusso NF. A cellular micro-RNA, let-7i, regulates toll-like receptor 4 expression and contributes to cholangiocyte immune responses against Cryptosporidium parvum infection. J Biol Chem. 2007;282:28929-38.

42. Zong Y, Wang H, Dong W, Quan X, Zhu H, Xu Y, Huang L, Ma C, Qin C. miR29C regulates BACE1 protein expression. Brain Res. 2011;1395:108-15.

43. Lee S-T, Chu K, Jung K-H, Kim JH, Huh J-Y, Yoon H, Park D-K, Lim J-Y, Kim J-M, Jeon D, Ryu H, Lee SK, Kim M, Roh J-K. miR-206 regulates brain-derived neurotrophic factor in Alzheimer disease model. Ann Neurol. 2012;72:269-77.

44. Tian N, Cao Z, Zhang Y. MiR-206 decreases brain-derived neurotrophic factor levels in a transgenic mouse model of Alzheimer's disease. Neurosci Bull. 2014;30:191-7.

45. Ren J, Zhang J, Xu N, Han G, Geng Q, Song J, Li S, Zhao J, Chen H. Signature of circulating MicroRNAs As potential biomarkers in vulnerable coronary artery disease. PLoS One. 2013;8(12):e80738.

46. Villa C, Fenoglio C, De Riz M, Clerici F, Marcone A, Benussi L, Ghidoni R, Gallone S, Cortini F, Serpente M, Cantoni C, Fumagalli G, Boneschi FM, Cappa S, Binetti G, Franceschi M, Rainero I, Giordana MT, Mariani C, Bresolin N, Scarpini E, Galimberti D. Role of hnRNP-A1 and miR-590-3p in neuronal death: genetics and expression analysis in patients with Alzheimer disease and frontotemporal lobar degeneration. Rejuvenation Res. 2011;14:275-81.

47. Narasimhan M, Patel D, Vedpathak D, Rathinam M, Henderson G, Mahimainathan L. Identification of Novel microRNAs in Post-Transcriptional Control of Nrf2 Expression and Redox Homeostasis in Neuronal, SH-SY5Y Cells. PLoS One. 2012;7(12):e51111.

48. Kumar P, Dezso Z, MacKenzie C, Oestreicher J, Agoulnik S, Byrne M, Bernier F, Yanagimachi M, Aoshima K, Oda Y. Circulating miRNA biomarkers for Alzheimer's disease. PLoS One. 2013;8:e69807.

49. Wang T, Chen K, Li H, Dong S, Su N, Liu Y, Cheng Y, Dai J, Yang C, Xiao S. The Feasibility of Utilizing Plasma MiRNA107 and BACE1 Messenger RNA Gene Expression for Clinical Diagnosis of Amnestic Mild Cognitive Impairment. J Clin Psychiatry. 2015;76(2):135-41.

50. Leidinger $P$, Backes $C$, Deutscher S, Schmitt K, Mueller SC, Frese K, Haas J, Ruprecht K, Paul F, Stähler C, Lang CJ, Meder B, Bartfai T, Meese E, Keller A. A blood based 12-miRNA signature of Alzheimer disease patients. Genome Biol. 2013;14:R78.

\section{Submit your next manuscript to BioMed Central and we will help you at every step:}

- We accept pre-submission inquiries

- Our selector tool helps you to find the most relevant journal

- We provide round the clock customer support

- Convenient online submission

- Thorough peer review

- Inclusion in PubMed and all major indexing services

- Maximum visibility for your research

Submit your manuscript at www.biomedcentral.com/submit 